\title{
Arthur Barsky: «Je größer der medizinische Fortschritt, desto kränker fühlen sich die Menschen»
}

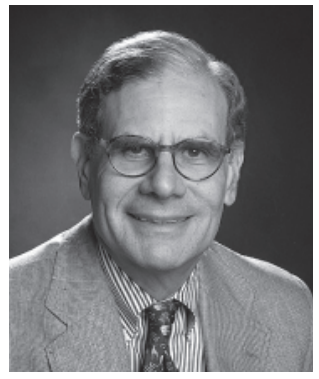

Prof. Arthur Barsky (M.D.) ist Professor für Psychiatrie an der Harvard Medical School und Leiter der psychiatrischen Forschungseinheit am Brigham and Women's Hospital in Boston, MA, USA. Seit vielen Jahren veröffentlicht er Arbeiten zu den Themen Krankheitsangst, Inanspruchnahme medizinischer Leistungen sowie Verhaltenstherapie bei körperlichen Beschwerden und Hypochondrie. Seine zahlreichen Arbeiten erschienen in Zeitschriften wie dem "New England Journal of Medicine", dem "Journal of the American Association (JAMA)" und anderen. Das Interview führte Winfried Rief, Professor für Klinische Psychologie und Psychotherapie an der Universität Marburg.

Lieber Arthur Barsky, vielen Dank für deine Bereitschaft, am Interview teilzunehmen. Du hast bereits 1988 eine Arbeit veröffentlicht mit dem Titel 'The paradox of health'. In dieser Arbeit führt du aus, dass zwar einerseits der medizinische Fortschritt immer mehr zunimmt, sich aber andererseits die Menschen trotzdem nicht gesünder fühlen. Wie lässt sich dieses Paradox erklären?

Arthur Barsky: Stimmt, es handelt sich hier um paradox anmutende Phänomene, die sich vermutlich im Sinne eines Teufelskreises gegenseitig aufschaukeln. Der medizinische Fortschritt hat zu vielen bahnbrechenden Erkenntnissen geführt. Man denke nur an die Möglichkeiten der Bildgebung, der genauen Erfassung von molekularen Vorgängen im Körper, mikrochirurgische Maßnahmen und vieles mehr. In den Medien wird ständig über neue medizinische Erfolge und neue Behandlungsmöglichkeiten berichtet. Die durchschnittliche Lebenserwartung ist in den letzten Jahrzehnten deutlich gestiegen. Dies scheint jedoch bei immer mehr Menschen zu der Erwartung beizutragen, alle Krankheiten seien behandelbar und alle körperlichen Missempfindungen benötigten ärztliche Interventionen. Während somit einerseits die medizinischen Möglichkeiten deutlich zugenommen haben, sind - fast noch dramatischer - die persönlichen Erwartungen an das Gesundheitssystem gewachsen. Oft erwarten Menschen zu viel bzw. unrealistische Erfolge von der Medizin.
Welche psychologischen Folgen könnte es haben, wenn Menschen zu hohe Erwartungen an die Behandelbarkeit körperlicher Beschwerden haben?

Arthur Barsky: Ich habe den Eindruck, dass viele Menschen durch ihre hohen Erwartungen an die Behandelbarkeit von Erkrankungen auch schneller mit ihrem Arzt unzufrieden sind, ihn häufiger wechseln, zusätzliche Meinungen von anderen Ärzten einholen etc. Es scheint sich allgemein das Verständnis herausgebildet zu haben, dass alle Krankheiten behandelbar wären und jede körperliche Missempfindung Zeichen einer Erkrankung ist. Vermutlich hat in diesem $\mathrm{Zu}-$ sammenhang auch die Selbstbeobachtung bezüglich körperlicher Missempfindungen zugenommen.

Im Zusammenhang mit Selbstbeobachtung sprichst du immer wieder von der "somatosensorischen Verstärkung». Was ist damit gemeint?

Arthur Barsky: Mit somatosensorischer Verstärkung meinen wir, dass Menschen unter Stress oder ängstliche Menschen dazu neigen, ihren Körper verstärkt zu beobachten. Durch diese erhöhte Selbstbeobachtung verändert sich die Wahrnehmung möglicher Körpermissempfindungen. Es kommt zu einem Verstärkungsprozess, so dass die Intensität dieser Missempfindungen als höher erlebt wird. Dies erhöht auch die

\begin{tabular}{llll}
\hline KARGER & ๑ 2007 S. Karger GmbH, Freiburg & & $\begin{array}{l}\text { Prof. Dr. Winfried Rief } \\
\text { Philipps-Universität Marburg }\end{array}$ \\
Fax +49 7614520714 & Accessible online at: & Klinische Psychologie und Psychotherapie \\
$\begin{array}{l}\text { E-mail Information@Karger.de } \\
\text { www.karger.com }\end{array}$ & www.karger.com/ver & Gutenbergstr. 18, 35032 Marburg, Deutschland \\
& & Tel. +49 6421 282-3657, Fax -8904 \\
& & E-mail rief@staff.uni-marburg.de
\end{tabular}


Wahrscheinlichkeit, dass Körpermissempfindungen falsch bewertet werden. Daraus ergibt sich auch ein Zusammenhang zu überhöhten Erwartungen an die Behandelbarkeit von körperlichen Erkrankungen. Je höher die Erwartungen an die Medizin sind, desto stärker ist vermutlich auch der Prozess der somatosensorischen Verstärkung ausgeprägt.

Du hast das Konzept der somatosensorischen Verstärkung vor allem im Kontext der Hypochondrie untersucht. Ist es nicht naheliegend, dass auch bei Menschen mit verschiedenen körperlichen Erkrankungen solche Sensibilisierungsprozesse der Wahrnehmung stattfinden?

Arthur Barsky: Das ist schon richtig. Bei Menschen mit Hypochondrie können wir davon ausgehen, dass dieses Merkmal der somatosensorischen Verstärkung fast immer vorliegt. Demgegenüber kann es bei Menschen mit körperlichen Erkrankungen einen komplizierenden Faktor darstellen, der jedoch nicht bei allen betroffenen Personen vorliegen muss. Trotzdem spielen solche Verarbeitungsprozesse auch bei körperlichen Erkrankungen eine große Rolle. Menschen, die grundsätzlich zu einer solchen Verarbeitung von Körperwahrnehmungen neigen, werden sich umso schwerer tun, mit einer körperlichen Erkrankung zurechtzukommen. Oft zeigt sich bei Menschen mit ausgeprägter Neigung zur somatosensorischen Verstärkung, dass sie medizinische Erklärungen nur schlecht annehmen können und medizinische Rückversicherungen keinen beruhigenden Effekt haben. Dies wiederum erhöht die Unzufriedenheit mit dem Arzt.

Wäre es nicht auch vorstellbar, dass körperliche Krankheiten eine erhöhte Neigung zu somatosensorischer Verstärkung auslösen? Oder gehst du davon aus, dass somatosensorische Verstärkung ein Persönlichkeitszug ist, der grundsätzlich vor der körperlichen Erkrankung vorliegt?

Arthur Barsky: Bei den meisten Patienten, mit denen wir zu tun haben, scheint es sich eher um einen Persönlichkeitszug zu handeln, der schon länger besteht. Allerdings wäre es interessant, näher zu untersuchen, ob nicht in manchen Fällen erst eine körperliche Erkrankung dazu führt, dass neue Verarbeitungsstile im Sinne der somatosensorischen Verstärkung entstehen.

Wie gut sind angehende Ärztinnen und Ärzte darauf vorbereitet, mit Patienten umzugehen, die starke Gesundheitsängste haben und zu dysfunktionalen Verarbeitungsmechanismen neigen, wenn sie körperliche Beschwerden haben?

Arthur Barsky: Ohne Frage besteht hier ein großes Manko. Viele Ärzte definieren ihre Rolle als Personen, die schwere körperliche Erkrankungen erkennen und behandeln sollen. In den meisten Ländern setzt auch das Medizinstudium diesen Schwerpunkt. Dem steht gegenüber, dass in der Allgemeinbe- völkerung die Rolle eines Arztes oft anders definiert wird. Menschen gehen mit verschiedensten körperlichen und seelischen Beschwerden zum Arzt und wünschen sich nicht nur, dass er oder sie eine schwere körperliche Erkrankung ausschließt. Viele Ärzte haben jedoch das Selbstverständnis, dass es nicht ihre Aufgabe ist, sich um allgemeines Wohlbefinden zu kümmern. Offensichtlich benötigen wir eine philosophische Neudefinition der Arztrolle. Vor allem der Hausarzt, aber auch die Fachärzte sollen sich nicht nur über den Umgang mit schweren körperlichen Erkrankungen definieren, sondern sich auch als Ansprechpartner für körperliche und seelische Gesundheit verstehen.

Siehst du Veränderungsmöglichkeiten im Medizinstudium, um auf diese erweiterte Arztrolle vorzubereiten?

Arthur Barsky: Man darf nicht pessimistisch sein. Es gibt wirklich Hinweise auf Verbesserungen. In den USA wird diese Erweiterung der Rollendefinition von Ärzten ernsthaft diskutiert, und es gibt bereits erste Anzeichen, dass dies in der Arztausbildung stärker berücksichtigt wird.

Bei vielen Menschen handelt es sich nicht nur um leichte Gesundheitsängste, sondern um das vollausgebildete Störungsbild der Hypochondrie. Ihr habt in einer Arbeit im «New England Journal of Medicine» eine Intervention vorgestellt, die 6 Therapiestunden für Personen mit Hypochondrie umfasst. Reichen 6 Stunden wirklich aus, um Personen mit dem Vollbild einer Hypochondrie zu behandeln?

Arthur Barsky: Es stimmt, dass dies eine Schwachstelle unserer Arbeit war. Wir waren in diesem Punkt etwas zu optimistisch. Ich würde zwischenzeitlich zustimmen, dass 6 Stunden für die meisten unserer Patienten zu wenig war. Aus meiner Sicht ist eine sinnvolle Behandlungsdauer für Hypochondrie 10 Therapiestunden lang und sollte mit einigen zusätzlichen Auffrischungssitzungen zu einer weiteren Stabilisierung des Gesundheitszustands beitragen. In unseren Interventionen werden sehr viele Informationen vermittelt bzw. mit den Patienten erarbeitet, so dass auch dafür ausreichend Zeit zu veranschlagen ist.

Was würdest du als Hauptinhalte einer kognitiven Verhaltenstherapie der Hypochondrie bezeichnen?

Arthur Barsky: Im Vordergrund stehen sicherlich die Gedanken und Bewertungsprozesse einer Person, vor allem ihre Vorstellungen über Gesundheit und Krankheit, über die Ursache von körperlichen Beschwerden und so weiter. Es ist aber genauso wichtig, die Aufmerksamkeitsstile zu beachten sowie die erhöhte Hypervigilanz, die bei vielen vorliegt. Wer ständig angespannt darauf wartet, neue Zeichen von Erkrankungen bei sich zu entdecken, kann eine Hypochondrie kaum bewältigen. Eine Veränderung der Aufmerksamkeitsprozesse muss in 
der Therapie oft direkt erarbeitet werden. Man darf nicht erwarten, dass diese sich durch andere Interventionen automatisch ergibt. Neben diesen Hauptfaktoren der Therapie sollte jedoch auch die Verbindung zu anderen Lebensbedingungen hergestellt werden, also z.B. zu Stressfaktoren, die positiv oder negativ auf die Symptomatik einwirken. Wenn diese Interventionen berücksichtigt werden, ist die Hypochondrie erfolgreich behandelbar.

\section{Gibt es auch Selbsthilfemöglichkeiten bei Hypochondrie?}

Arthur Barsky: Wir haben ein Büchlein herausgegeben für Menschen, die unklare körperliche Beschwerden und erhöhte Krankheitsängste haben. Die Erfahrungen damit sind sehr gut. Trotzdem gehe ich davon aus, dass beim Vollbild einer Hypochondrie und entsprechender Chronifizierung in der Regel eine ausführliche Therapie notwendig ist.
Du hast daraufhin gewiesen, dass mit zunehmender Lebenserwartung durch die Erfolge der Medizin auch die Unzufriedenheit mit klassischen medizinischen Ansätzen, mit Ärzten und medizinischen Interventionen angestiegen ist. Bedeutet dies, dass in Zukunft psychologischen Ansätzen ein höherer Stellenwert zukommen wird, um die Erwartungen und Krankheitsängste von Personen besser zu berücksichtigen?

Arthur Barsky: Das halte ich für gut möglich. Durch die Fokussierung medizinischer Entwicklungen auf organische Bereiche ist die psychologische Betreuung durch unsere Gesundheitsexperten in den Hintergrund geraten. Dies ist jedoch ein ausgesprochenes Bedürfnis vieler unserer Patienten.

Vielen Dank für das Gespräch! 